\title{
Mitochondrial Phylogeny and Population Structure of Pakistani Dromedary Camel (Camelus dromedarius)
}

\author{
Abdul Fatih ${ }^{1}$, Abdul Wajid ${ }^{2}$, Muhammad Masood Tariq ${ }^{1 *}$, Masroor Ahmad Bajwa ${ }^{1}$, Masroor Ellahi Babar ${ }^{3}$, Pamela \\ Anna Burger ${ }^{4}$ and Tanveer Hussain ${ }^{*}$ \\ ${ }^{1}$ Center for Advanced Studies in Vaccinology and Biotechnology, University of Balochistan, Pakistan \\ ${ }^{2}$ Department of Biotechnology, Virtual University of Pakistan \\ ${ }^{3}$ University of Agriculture, D.I. Khan, Khyber-Pakhtunkhwa, Pakistan \\ ${ }^{4}$ Research Institute of Wildlife Ecology, University of Veterinary Medicine Vienna, Austria \\ ${ }^{5}$ Department of Molecular Biology, Virtual University of Pakistan \\ *For correspondence: tanveer.hussain@vu.edu.pk \\ Received 23 November 2020; Accepted 22 January 2021; Published 16 April 2021
}

\begin{abstract}
Dromedary camels (Camelus dromedarius) are one of the most important livestock species mainly used for milk and meat production in semi-arid and hot-desert expanses of the Arabian-Peninsula, Africa, and Southwest Asia. This study investigated the genetic diversity and population structure within and between eight dromedary camel breeds $(n=210)$ inhabiting Balochistan province, Pakistan, by mitochondrial cytochrome b $(C y t b)$. Sequences (1140 bp) analysis showed a total of 18 variable sites resulting in 16 haplotypes. The average haplotype and nucleotide diversities were $\mathrm{Hd}=0.484 \pm 0.051$ and $\pi=$ 0.00272 respectively. Phylogenetic analysis showed different clusters for camelids. The neutrality tests did not support the population demographic expansion for these camel breeds. Based on these results, we suggest that an imperative genetic management and breeding strategies are required for the effective conservation of this species. (C) 2021 Friends Science Publishers
\end{abstract}

Keywords: $C y t$ b; Haplotypes; Phylogeny; Dromedary camels; Balochistan

\section{Introduction}

The genus Camelus is an interesting species inhabiting cold and hot deserts and as well as high and low altitude worldwide. The family Camelidae is divided into two groups, New-World (Lamini) and Old-World (Camelini) camels. Within Camelini, two domesticated species are described including the single-humped dromedary or Arabian camel, (Camelus dromedaries) found in MiddleEast, Horn of Africa and Southeast of Asia (Bahbahani et al. 2019) and the two-humped Bactrian camel (Camelus bacterianus) found in Central and Eastern-Asia, as well as the critically endangered two-humped wild camel (Camelus ferus) distributed in remote areas of the Gobi and Taklamakan deserts in Mongolia and Northwest China (Yadamsuren et al. 2019; Fitak et al. 2020).

The world population of the camel is estimated at around 35 million with the majority $(90 \%)$ are the dromedaries (FAO 2019). In Pakistan, the population of the camel is estimated to be around 1.0 million distributed in arid, semi-arid, and desert areas of the country with 20 different recognized breeds. They have been providing rich organic milk, meat, hides, wool, dancing/racing animal, and transport facility provider. Camels are an important part of the country's economy and have a significant role in the socio-economic uplift of the local community. The hardy nature of camel with wonderful physiological characteristics has made it the best-suited animal for draft purposes in the climatically harsh and inaccessible regions (Chen et al. 2019).

Dromedaries are one of the major pastoral domestic species in Pakistan along with few herds of Bactrian camels found in Northern areas. Balochistan shares a total of $41 \%$ of dromedary camels in Pakistan, followed by Punjab (22\%), Sindh (30\%) and Khyber Pakhtunkhawa (7\%) respectively. Balochistan is situated in the southwestern part of Pakistan in a desert belt between $25^{\circ} \mathrm{N}$ to $32^{\circ} \mathrm{N}$ latitude and $60^{\circ} \mathrm{E}$ to $72^{\circ} \mathrm{E}$ longitude. Balochistan contains mostly arid and semi-arid areas, deserts and mountainous regions. Camels are an important animal in the province's livestock herds and play a vital role in the economy of rural pastoral communities. Overall, the camel is still a neglected species in Pakistan and has not given proper attention by scientists and researchers. Comprehensive information on the

To cite this paper: Fatih A, A Wajid, MM Tariq, MA Bajwa, ME Babar, PA Burger, T Hussain (2021). Mitochondrial phylogeny and population structure of Pakistani dromedary camel (Camelus dromedarius). Intl J Agric Biol 25:981-984 
population structure and diversity found in dromedaries from Balochistan province is still missing. To fill this gap and to understand the phylogenetic relationship between populations, we investigated the genetic diversity among eight camel breeds using mitochondrial $C y t \quad b$ gene sequences. With this study we aim to provide first genetic data for genetic-based breed management in dromedaries of Balochistan province.

\section{Materials and Methods}

In total, we sampled 210 animals from eight dromedary camel breeds in geographically different districts of Balochistan province (Fig. 1), including the Brahvi, Kharani, Kachhi, Mukrani, Roadbari, Pishin, Lassi and Kohi well adapted to hot, arid and cold, dry environments of Balochistan. The blood samples were collected in EDTAcontaining vacutainer-tubes and were carried on ice-box to Animal Genomic Laboratories, Virtual University of Pakistan. The DNA was isolated from the blood using revised phenol/chloroform procedure previously described by Wajid et al. (2014). The extracted DNA samples were quantified using the Nanodrop spectrophotometer (Thermoscientific, U.S.A.) and stored at $-20^{\circ} \mathrm{C}$ until further use. The complete $C y t b$ gene was amplified and sequenced using three-overlapping primers previously reported by Babar et al. (2015). PCR amplification was carried out in a final volume of $30 \mu \mathrm{L}$ mixture contained genomic DNA (20 ng) $2 \mu \mathrm{L}$, forward and reverse primers (10 pmol) $1.5 \mu \mathrm{L}, \mathrm{dNTPs}$ (2.5 $\mathrm{m} M) 2 \mu \mathrm{L}$, reaction-buffer (10X) $3.5 \mu \mathrm{L}, \mathrm{MgCl}_{2}(1.5$ $\mathrm{m} M$ ) $3.5 \mu \mathrm{L}$, Taq DNA-polymerase (5Unit) $0.5 \mu \mathrm{L}$ (Thermo-scientific) and $\mathrm{H}_{2} \mathrm{O} 17 \mu \mathrm{L}$. The amplification was carried out in BioRad Thermos-cycler using initial denaturation of $95^{\circ} \mathrm{C}$ for $5 \mathrm{~min}$ followed by 33 cycles of denaturation at $95^{\circ} \mathrm{C}$ for the $30 \mathrm{~s}$, annealing at $60^{\circ} \mathrm{C}$ for $30 \mathrm{~s}$ and extension at $72^{\circ} \mathrm{C}$ for $1 \mathrm{~min}$ and then with a final extension at $72^{\circ} \mathrm{C}$ for $7 \mathrm{~min}$. The purified $C y t b$ gene PCR products were sequenced by $\mathrm{ABI} 3130$ automated-sequencer (ABI, Inc., Foster City, C.A.). A Maximum Likelihood method phylogenetic tree was constructed to investigate the evolutionary relationship of the studied camel breeds with other dromedary, and Bactrian camel breeds using MEGAv6 software. DnaSP v. 5 program was used for conducting neutrality tests, i.e., Tajima's D and Fu and Li's $F$ to detect a deviation from neutrality, and to calculate within species diversity parameters, e.g., number of haplotypes, nucleotide and haplotype diversities. The complete $C y t b$ haplotypes $(n=16)$ obtained in this study were submitted to GenBank and are available under accession number MT578032 to MT578047.

\section{Results}

The mtDNA sequence analysis of $1140 \mathrm{bp}$ in 210 animals showed a total of 18 variable sites resulting in 16 different haplotypes that demonstrated a moderate mtDNA genetic diversity in the Pakistani camel populations. The detail of the number of variations in each breed, haplotype, haplotype diversities, and nucleotide diversities are shown in Table 1. Among the studied camel breeds, the average haplotype and nucleotide diversities were $\mathrm{Hd}=0.484 \pm 0.051$ and $\pi=$ 0.00272 respectively. Highest number of haplotypes $(n=3)$ was detected in Pishin and Kharani dromedaries while only a single haplotype was identified in Kachhi and Rodbari breeds, respectively. The haplotype diversity ranged from $\mathrm{Hd}=0.500 \pm 0.048$ in the three populations Brahvi, Kohi, and Lassi to $\mathrm{Hd}=0.690 \pm 0.023$ in Pishin and Kharani (Table 1). Nucleotide diversity ranged from $\pi=0.00044$ (Lassi) to $\pi=0.00242$ (Kharani). The haplotypes identified in camel populations under study were unique to each population, except Markrani and Rodbari shared one haplotype; this is a likely crossbreeding as geographically nearest neighbors.

The Rodbari camel breeds showed highest genetic relatedness $(0.1 \%)$ with other two Makrani and Lassi camel breeds. Among all studied breeds, Kachhi was divergent camel exhibited $1.3 \%$ genetic distance from Makrani and Kharani camel breeds. A Maximum Likelihood method phylogenetic tree was constructed to investigate the evolutionary history of the studied camel breeds with other dromedary and Bactrian camels from Arabian Peninsula, Iran, Ethopia, Kenya, Russia, China and Mongolia. The result clearly exhibited two distinct clades, dromedary and Bactrian. The Bactrian camels further divided into two different lineages, the domestic and wild animals (Fig. 2). Two classical tests were employed to detect deviation from neutrality. We obtained positive values of Tajima's D and $\mathrm{Fu}$ and Li's F tests in all studied camel breeds, indicative of balancing selection or a population subdivision event, but the result was statistically significant $(P<0.05)$ only in three camel breeds.

\section{Discussion}

In this study, we investigated the genetic diversity and phylogenetic relationship among 210 dromedaries belonging to eight camel breeds reared in Baluchistan province using complete mitochondrial Cyto b amplification. Retrieving this genetic information is a first indispensable step to facilitate the breed conservation program in an effective and reminiscent way. Camel ecotype is not well defined in Pakistan and very little information is available on genetic-studies in camels found all over the country. Hence, the molecular studies on genetic diversity in this specie can facilitate the development of national camel-breeding program to establish an effective strategy for the conservation of these important genetic resources.

$C y t b$ is a highly conserved region of mitochondrial DNA (mtDNA) genome used for studying species classification and detection of phylogenetic relations among diverse mammalian species. 
Phylogeny of Pakistani Camel Breeds / Intl J Agric Biol, Vol 25, No 5, 2021

Table 1: The genetic diversity analysis of studied camel populations

\begin{tabular}{|c|c|c|c|c|c|c|c|c|c|}
\hline Species & Samples & Detected variations & $\mathrm{H}$ & Haplotype diversity (Hd) & Nucleotide diversity (Pi) & $\mathrm{K}$ & Tajima's D & $P$ value & Fu and Li's F \\
\hline Pishin & 30 & 2 & 3 & $0.690 \pm 0.023$ & 0.00081 & 0.91954 & 1.68264 & $P>0.10$ & $1.21673 \mathrm{P}>0.10$ \\
\hline Brahvi & 25 & 3 & 2 & $0.500 \pm 0.048$ & 0.00132 & 1.50000 & 2.20530 & $\mathrm{P}<0.05^{*}$ & $1.52520 \mathrm{P}<0.05 *$ \\
\hline Kachhi & 25 & 0 & 1 & 0.000 & 0.00000 & 0.00000 & 0.00000 & 0.00 & 0.00000 \\
\hline Kharani & 30 & 6 & 3 & $0.690 \pm 0.023$ & 0.00242 & 2.75900 & 2.34410 & $\mathrm{P}<0.05^{*}$ & $1.80694 \mathrm{P}<0.05^{*}$ \\
\hline Kohi & 25 & 2 & 2 & $0.500 \pm 0.048$ & 0.00088 & 1.00000 & 1.92637 & $\mathrm{P}>0.10$ & $1.30700 \mathrm{P}>0.10$ \\
\hline Lassi & 25 & 1 & 2 & $0.500 \pm 0.048$ & 0.00044 & 0.50000 & 1.47274 & $P>0.10$ & $0.97500 \mathrm{P}>0.10$ \\
\hline Makrani & 25 & 4 & 2 & $0.513 \pm 0.037$ & 0.00180 & 2.05300 & 2.53549 & $\mathrm{P}<0.01 *$ & $1.73014 \mathrm{P}<0.05^{*}$ \\
\hline Rodbari & 25 & 0 & 1 & 0.000 & 0.00000 & 0.00000 & 0.00000 & 0.00 & 0.00000 \\
\hline
\end{tabular}

H: Haplotype

$\mathrm{K}$ : the average number of nucleotide differences

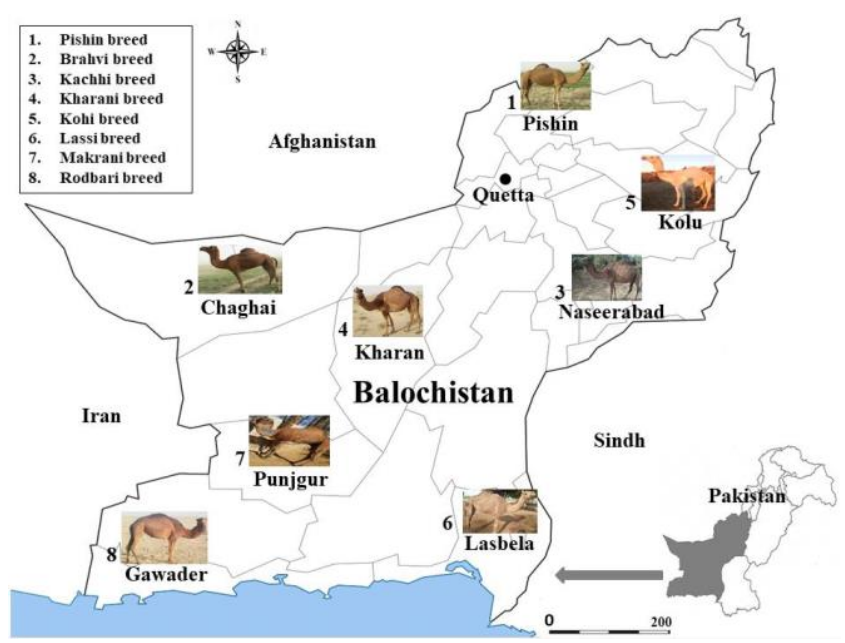

Fig. 1: Geographical locations of the samples used in this study

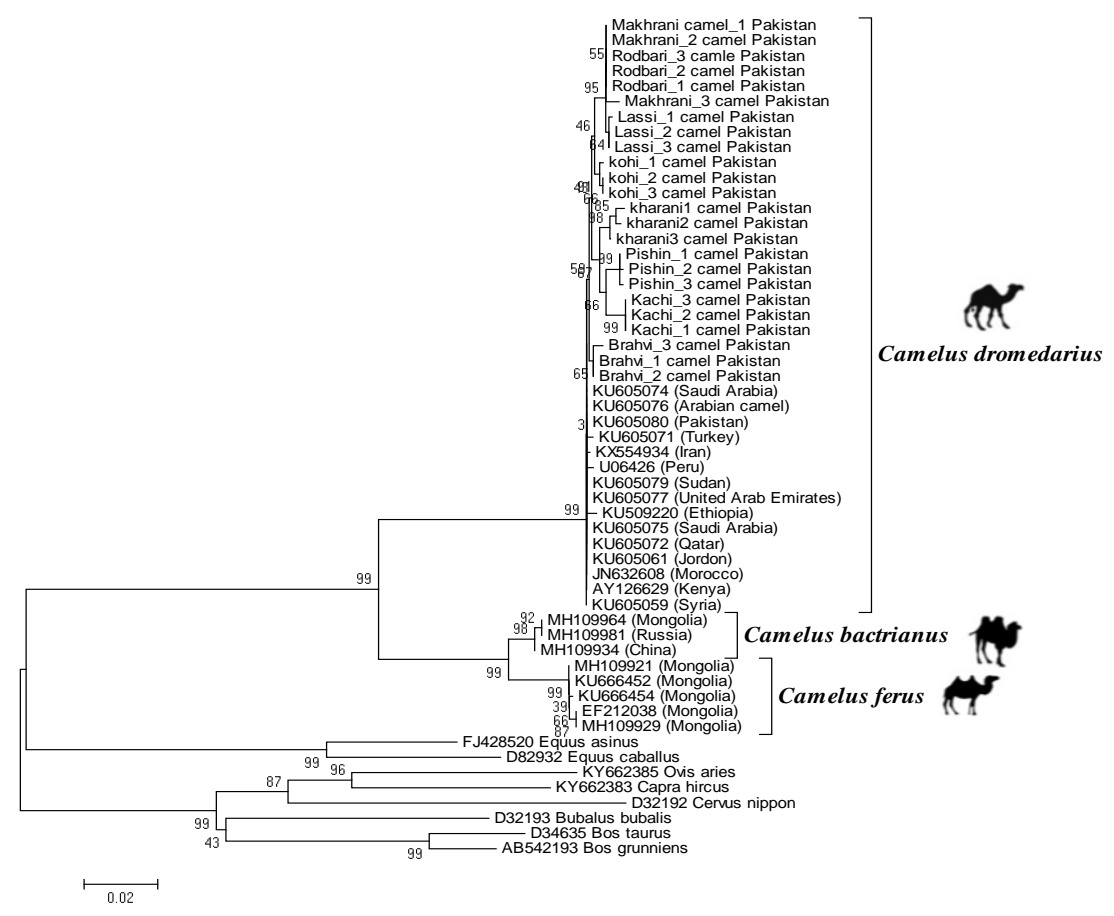

Fig. 2: Maximum-Likelihood method phylogenetic tree inferred from Cyto $b$ gene sequences

The partial and complete $C y t b$ has been used for detection of genetic conservation and phylogenetic relationship among Bactrian camel (Ming et al. 2016), dromedaries (Babar et al. 2015; Legesse et al. 2018) and other livestock species (Hussain et al. 2018). In this study, complete $C y$ to $b$ gene has been used for comparing genetic 
diversity between eight camel breeds rearing in different geographical regions of Balochistan. The breeds showed low differences in their genetic diversity. The haplotypes identified were unique to each breed except the two camel breeds except Markrani and Rodbari shared one haplotype. The uniqueness of the haplotypes identified in the studied camel populations generally suggests the haplotypes specific to a geographic region and low level of crossbreeding.

Two classical tests were employed to detect deviation from neutrality. The positive $\mathrm{D}$ values indicate an excess of intermediate frequency alleles and may suggest balancing selection or population contraction. Neutrality tests were not performed on Kachhi and Rodbari camel breeds, because these species had no genetic variation in the $C y t b$ gene. The neutrality tests showed no population demographic expansion for the eight studied camel populations. Phylogenetic analysis was performed based on the complete $C y t b$ gene and showed different clusters for camelids. The phylogeny of the $C y t b$ gene was consistent with known phylogeny for the ruminant classification based on other genes (Iqbal et al. 2020).

\section{Conclusion}

This study provides initial information on the genetic diversity of camel populations reared in Balochistan province. The studied camel populations exhibited low levels of haplotype and nucleotide diversities, which may be due to the small effective population size or many years inbreeding that may further diminish the genetic diversity in these camel populations. Indeed, the camels are well adaptable domestic animals to the harsh ecosystems where these animals are essential for the economy and food security of local communities. We suggest based on these results, an imperative genetic management and breeding strategies are required for effective conservation of this species.

\section{Acknowlegment}

We would like to thank to the farmers and the staffs of the Livestock \& Dairy Development Department (L\&DD) of Balochistan province, helped us in samples collection. Laboratory work was performed under the HEC grant (NRPU-9336) provided to Dr. Tanveer Hussain

\section{Author Contributions}

AF, AW and MTM designed and perceived the experiment, $\mathrm{AF}, \mathrm{MEB}$ and AW execute the experiment, $\mathrm{AF}$ and $\mathrm{TH}$ collected the samples and $\mathrm{AF}$ and $\mathrm{TH}$ helped in data analysis, AW wrote the manuscript.

\section{Conflicts of Interest}

All authors declare no conflicts of interest.

\section{Data Availability}

Data presented in this study are available on fair request to the corresponding author.

\section{Ethics Approval}

The experiments were carried out in accordance with the guidelines issued by the Ethical Committee of University of Balochistan, Quetta, Pakistan.

\section{References}

Babar ME, T Hussain, A Wajid, A Nawaz, A Nadeem, SA Shah, MA Shahid, N Ahmad, K Javed, M Abdullah (2015). Mitochondrial cytochrome-b and D-loop sequence-based genetic diversity in Mareecha and Bareela camel breeds of Pakistan. J Anim Plant Sci 25:591-594

Bahbahani H, HH Musa, D Wragg, ES Shuiep, F Almathen, O Hanotte (2019). Genome diversity and signatures of selection for production and performance traits in dromedary camels. Front Genet 10; Article 893

Chen SG, J Lic, F Zhang, B Xiao, JM Hu, YQ Cui, M Hofreiter, XD Hou, GL Sheng, XL Lai, JX Yuan (2019). Different maternal lineages revealed by ancient mitochondrial genome of Camelus bactrianus from China. Mitochondrial DNA A DNA Mapp Seq Anal 30:786-793

FAO (2019). The Food and Agriculture Organization of the United Nations. Italy: FAOSTAT

Fitak RR, E Mohandesan, J Corander, A Yadamsuren, B Chuluunbat, O Abdelhadi, A Raziq, P Nagy, C Walzer, B Faye, PA Burger (2020). Genomic signatures of domestication in Old World camels. Commun Biol 3:316-325

Hussain T, ME Babar, MD Donato, A Wajid, A Nadeem, Z Ahmad, WA Khan, SO Peters, IG Imumorin (2018). Phylogeny of Pakistani Cattle Breeds using Mitochondrial Cytochrom-b Gene. Pak J Zool 50:2029-2035

Iqbal LN, Q Ain, A Wajid, A Mohiuddin, TM Yousaf, N Hussain, S Sherzada, F Manzoor, T Hussain, ME Babar (2020). Detection of genetic variations in pattern recognition receptors (PRRs) gene of Tharparkar cattle breed. Pak Vet J 40:400-402

Legesse YW, CD Dunn, MR Mauldin, N Ordonez-Garza, GR Rowden, YM Gebre, MY Kurtu, SM Ali, WD Whibesilassie, M Ballou, M Tefera, G Perry, RD Bradley (2018). Morphometric and genetic variation in 8 breeds of Ethiopian camels (Camelus dromedarius). J Anim Sci 96:4925-4934

Ming L, L Yi, FC Guo, G Siriguleng, J Jirimutu (2016). Molecular phylogeny of the Bactrian camel based on mitochondrial Cytochrome-b gene sequences. Genet Mol Res 15:1-8

Wajid A, M Wasim, T Yaqub, S Firyal, M Tayyab, S Siddique, T Hussain (2014). Assessment of genetic diversity in Balochi and Rakhshani sheep Breeds of Balochistan using microsatellite DNA-markers. $J$ Anim Plant Sci 24:1348-1354

Yadamsuren A, O Daria, S Liu (2019). The seasonal distribution of wildcamels (Camelus ferus) in relation to changes of the environmental conditions in Mongolia. Open J Ecol 9:293-314 